\title{
What Happens in Peer-Support, Stays in Peer-Support: Software Architecture for Peer-Sourcing in Mental Health
}

\author{
Mahsa Honary \\ Department of Engineering \\ University of Cambridge, UK \\ mh2035@cam.ac.uk
}

\author{
Jaejoon Lee \\ School of Computing Sciences \\ University of East Anglia, UK \\ jaejoon.lee@uea.ac.uk
}

\author{
Christopher Bull \\ School of Computing and Communications \\ Lancaster University, $U K$ \\ c.bull@lancaster.ac.uk
}

\author{
Jiangtao Wang \\ School of Computing and Communications \\ Lancaster University, UK \\ jiangtao.wang@lancaster.ac.uk
}

\author{
Sumi Helal \\ School of Computing and Communications \\ Lancaster University, $U K$ \\ s.helal@lancaster.ac.uk
}

\begin{abstract}
Digital health technology utilizing wearables, IoT and mobile devices has been successfully applied in the monitoring of numerous diseases and conditions. However, intervention, in response to monitored data, is yet to benefit from technological support and continues to follow a traditional point-of-care delivery model by providers and health professionals. Mental health is an example of a critical health area in dire need for technology solutions to enable timely, effective and scalable interventions. This is especially the case with an increasing prevalence of mental health conditions and a declining capacity of the healthcare professional workforce. Numerous studies reveal the potential for peer support groups as an effective, scalable, cost-effective, firstline of response in mental health interventions. Peer support helps participants, at low and moderate risk, better understand their diseases or conditions and empowers them to take control of their own health. Peer support interactions also seems to inform health professionals with insights and intricate knowledge, making it effectively a learning health system. This paper proposes a software architecture to better enable "peer-sourcing". We present related work and show how the proposed architecture might draw similarity to and differences from crowd-sourcing architectures. We also present a study in which we interacted with service users (mental health patients) and mental healthcare professionals to better understand and elicit the key requirements for the software architecture.
\end{abstract}

Index Terms-peer-sourcing, architecture, microservices, mental health, peer-support

\section{INTRODUCTION}

"Feeling 'less than', isolated, rejected, feeling a loss of belonging as part of the society. Not worthy of any love or interaction, the world would be a better place without me" This description resonates real experiences of people suffering from mental health conditions, highlighting loneliness and lack of support as contributing triggers.

Mental health is a major public health concern, with 1 in 7 people suffering from one or more mental health conditions globally [1]. There is no permanent cure for mental health conditions, however, long-term wellbeing can be achieved through appropriate management and intervention [2]. For this, service users are required to continuously monitor their symptoms, which would then be used to facilitate a timely intervention [3].

There is an increase in the number of studies utilizing technology to facilitate automatic symptom monitoring as opposed to a traditional model of questionnaire assessment in consultations [2]. This new model of data acquisition has the potential to inform health professionals more effectively, thus enhancing the digital therapeutic delivery. For example, the Beiwe platform was developed and tested to predict relapse in patients with schizophrenia through "moment-by-moment quantification of the individual-level human phenotype insitu using data from smartphones and other personal digital devices" [4].

Current digital health architectures mainly focus on detection and visualization [5]. There is a distinct lack of any 'care delivery' components in current software architectures and, indeed, using technology to facilitate appropriate forms of intervention. In this sense, there is a requirement in digital health architecture for thinking about ways of connecting individuals to appropriate sources of support when changes in mental wellbeing are detected. An appropriate source of support needs to take into account a user's preference. For example, who and when to engage with in their care.

Peer support is a non-technical intervention that has been defined as "the provision of emotional, appraisal, and informational assistance by a created social network member who possesses experiential knowledge of a specific behavior or stressor and similar characteristics as the target population" [6]. There exists some evidence to support alternative interventions, such as peer support, which are effective in reducing symptoms and rehospitalization [7]. Peer support is a rising phenomenon and is used broadly in various health conditions, spanning from cancer [8], mental health [9], to 
groups facing social changes such as new mums [10] or informal caregivers of people with long-term health conditions [11]. Peer support is increasing in demand, however not many technologies utilize this form of support. We describe peer support in more detail in Section II-A

Designing the software architecture of a peer support system is non-trivial due to the following reasons. First, since no stateof-the-art work systematically presents the requirements, we need to extract the need comprehensively and identify unique requirements. Second, we need to design an appropriate software architecture to support the development of peer support systems, which can fully meet those elicited requirements.

In this paper, we propose a software architecture for sourcing peer support in mental health, through "peer-sourcing", and provide the following contributions:

- We present the needs of people experiencing suicide ideation with respect to the support they wish to receive through the use of a learning health system.

- We have elicited software requirements based on these findings.

- We present a software architecture based on those peersourcing requirements to support predictive digital health technologies.

- We demonstrate a use case scenario where the components have been developed to realize the feasibility of the design.

The remainder of this paper is organized as follows: Section III presents the related work with respect to software architecture for crowd-sourcing in the mental health domain. Section III covers the challenges in delivering timely support in the domain of mental health and our requirements gathered through the co-design activities we carried out with people with lived-experience of suicide ideation and mental healthcare professionals. Section IV presents the proposed design methodology of peer-sourcing to support predictive technologies in the mental health domain. Finally, we discuss our proposal and conclude this paper in Sections $\mathrm{V}$ and $\mathrm{VI}$

\section{BACKGROUND AND RELATED WORK}

\section{A. The Role of Peer-Support in Mental Health}

One of the challenges with the provision of remote mental health support, through the use of technology, is low adherence and engagement with digital interventions. A study by Mohr et al. shows that when users are offered a weekly 5-10 min telephone consultation with a coach, in addition to access to a web-based intervention, users showed better retention towards the intervention [12]. While this model shows promise in improving user engagement, there are a number of implementation barriers including the delivery cost and difficulties with scheduling a meeting between the two parties.

The support coming from people who have similar experiences can offer more authentic empathy and validation [13]. Peer-support practice in mental health encourages sharing information and lived-experiences that can lead to better management of recovery focused activities [14], and is shown to be effective in a wide range of mental health conditions [15]. Peer-support offered through community mental health services is increasingly accepted [16]. It has been reported that the number of services run for and by people with mental health conditions is more than double the number of services run by professionals in the US [17]. The healthcare professional services are also starting to recognize the value of peer-support, and employing people with past lived-experience of mental health within services to provide care and support to others [15]. There is a growing interest in learning peer counseling skills [18]. It is highlighted in the literature that peer-support can benefit both the person receiving help and the person providing support [19]. The benefit for the peersupporter is seen in the skills they build through this process, which could improve the social and occupational functioning of the peer-supporter [20]. Peer-support can be delivered faceto-face and in online settings such as social networking sites [21].

Online peer-support networks have shown to be successful in attracting a wide range of users in the mental health domain [9]. In a randomized controlled trial of Panoply, a peerto-peer web-based cognitive reappraisal program, compared with an online expressive writing platform, Panoply generated significantly more usage activity and greater user experience scores [22].

\section{B. Software Architectures in Mental Health}

There are many software projects for mental health, but few provide details of their software architectures and how they benefit the mental health domain. Whilst several of the software architectures described below encourage a move away from the traditional point-of-care delivery (e.g., by supporting self-management of service user's health), they do so only for monitoring. Intervention falls back on self-care or the traditional point-of-care delivery model.

1) Personal Health Systems: These type of systems provide personal health management through monitoring and reporting, typically using personal devices (e.g. smartphones, medical sensors).

One example of an architecture designed specifically for mental health is a mobile pervasive architecture for stress monitoring in a personal health system [23]. Tartarisco et al. present an architecture for smartphone and wearable sensors to monitor stress of individuals with an integrated clinical decision support system. They claim the novelty of this approach compared to similar systems is that it is "suitable for prolonged stress monitoring during normal activity", due to its passive monitoring.

Another personal health monitoring system proposed by Mouttham et al. extends a Service-Oriented Architecture (SOA) with event-driven architectural elements [24]. This architecture aims to seamlessly connect personal health monitoring with existing e-Health systems.

2) Clinical Decision Support Systems: A common type of software system (or component) used in healthcare. A clinical decision support system (CDSS) assists health professionals 
in their decision-making tasks by providing timely, additional information related to a given patient or health context.

Velickovski et al. detail the 4 main types of CDSS, including a comparison of their features [25]. The 4 types are: standalone, integrated (into a health information system or electronic health record), standard-based (decoupled, yet interoperable), and service-oriented. In their work, Velickovski et al. chose a Service-Oriented Architecture to integrate web services into existing health information system platforms for the "early detection and assessment of chronic obstructive pulmonary disease (COPD)".

Distributed CDSS architectures have also been explored [26]. El-Sappagh and El-Masri propose a distributed CDSS which is a collection of services (SOA) and incorporates Electronic Health Records (EHR). They also claim that "SOA has been widely adopted to solve the interoperability of the involved heterogeneous distributed EHR systems" [26].

Another example of a CDSS is the enhanced medical record for aging brain care (eMR-ABC) [27], which was created using Software as a Service (SaaS) and SOA architectural styles. This CDSS integrates a decision support system with electronic medical records for the management of people suffering from aging brain disorders, such as dementia.

3) Relevant architectures in other domains: Relevant software architectures exist in other domains, which have similar properties to already mentioned systems. For example, eventdriven architectures for decision support (e.g., in a traffic management system [28]) could be used by a CDSS where timeliness is a critical factor.

Systems for ambient assisted living (AAL) can be used to provide aspects of healthcare in the home. One such example uses a Service-Oriented Architecture to deliver e-health which detects both mental and physical problems [29].

Another software architecture model is provided for behavior change support systems (BCSS) [30]. This BCSS is used only by service-users for a weight loss self-management application. Whilst BCSS are not directly related to mental health, it is conceivable that they could form part of a mental health system, e.g. providing persuasive behavior change components in a stress monitoring and feedback application.

The SAMS project aimed to detect early signs of Alzheimer's disease through the collection and analysis of home computer usage [31]. The reported architecture focuses on the desktop data collection components, whilst the transmission of the data to the server uses a client-server model. This system focused on the detection of Alzheimer's disease, but did not provide any mechanisms for intervention.

Despite the numerous software systems in the mental health domain, there appears to be no exploration of software architectural styles to support care delivery at the intervention level, rather than just the detection level.

\section{Crowd-sourcing Systems}

The term "crowd-sourcing" was originally coined by Jeff Howe and Mark Robinson in [32] to describe how businesses were using the Internet to outsource work to the crowd.
The basic idea of crowd-sourcing is to leverage the power of a crowd to collaboratively complete a complicated task. In recent years, crowd-sourcing-based systems are widely used in many application domains, such as citizen science, software engineering, semantic web, and crowdfunding [33][38]. To support various crowd-sourcing-based applications, many software infrastructures (e.g., platforms, middlewares, or programming frameworks) has been proposed. For example, Amazon Mechanical Turk (MTurk) [39] is a crowd-sourcing platform that makes it easier for individuals and businesses to outsource their human intelligence tasks (e.g., labeling images for machine learning) to a distributed workforce who can perform these tasks virtually. MTurk also provides many APIs for creating and managing crowd-sourcing tasks. Both [40] and [41] proposed programming frameworks with development and runtime support for mobile crowdsensing applications, which can be used to collect location-based urban sensing data (e.g., traffic status, air quality, and so on). The authors in [42] proposed a service platform that enables human-subject studies (e.g., survey deliveries and management) through crowdsourcing. Epicollect [43] allows the creation of crowd-sourcing tasks specific to epidemiology and ecology.

The above software infrastructures or frameworks do provide good support for its target application domains. However, they cannot fully meet the requirements 1 in our peer-sourcing mental health support scenario due to the following reasons. First, traditional crowd-sourcing systems commonly follow a centralized architecture, in which service requesters and providers are well connected via a central control agent. In contrast, peer-sourcing-based support applications require a decentralized architecture, where all peers and other relevant stakeholders can be connected with matching demands in a peer-to-peer and ad-hoc manner. Second, service providers in crowd-sourcing systems are usually homogeneous in terms of the expertise and reliability in completing relevant tasks. On the contrary, peers in peer-sourcing systems consist of multiple stakeholders with heterogeneous attributes. For example, peers in our mental health support scenarios can be buddy peers like friends and family members, professional peers like mental healthcare professionals, and peer-sourcing organizers like UK National Health Service (NHS) support groups. All of these peers have different attributes in terms of task assignment and peer selection. Third, crowd-sourcing platforms usually possess a large number of candidate service providers with low urgency requirements, while peer-sourcing often suffers from the insufficiency of service providers meanwhile with higher urgent requirement (e.g., the case of suicide intervention). Last but not the least, synchronous interactions between the requester and supporter are required in peer-sourcing, but there is no such need for this in crowd-sourcing. In summary, state-of-the-art crowd-sourcing architectures cannot be directly adopted to peer-sourcing applications due to the differences summarized in Table [1.

\footnotetext{
${ }^{1}$ Details of Peer-Sourcing requirements are discussed in Section III-E
} 
TABLE I

TYPICAL PROPERTIES OF CROWD-SOURCING VS. PEER-SOURCING

\begin{tabular}{ll}
\hline Crowd-sourcing & Peer-Sourcing \\
\hline Centralized & De-centralized \\
Homogeneous & Heterogeneous \\
Sufficient candidates & Insufficient candidates \\
Lower task urgency & Higher task urgency \\
Asynchronous interactions & Synchronous interactions \\
\hline
\end{tabular}

\section{REQUiREMENTS FOR PEER SUPPORT}

As shown in Section II-A, peer support plays an important and promising role as an alternative intervention in minor to mild mental health conditions. There is an important need to provide systematized capabilities for mental health care delivery through peer support. It alleviates pressures on the overstretched health system, delivers authentic support from people with similar experiences, and can lead to better management of mental health conditions, among other benefits.

Whilst there are no existing software architectures to support care delivery in mental health specifically, providing peer support seems to bear a similarity to how crowd-sourcing systems work, though with some distinct differences (see Table II. Crowd-sourcing is inherently limited when it comes to sourcing peer support, which is discussed later (Section $\mathrm{V}$ ). To that end, we endeavored to propose a new software architecture for peer-sourcing, by first eliciting requirements through a service user study conducted within the SPACE project.

\section{A. SPACE: A Learning Health System}

This research came out of the "Suicide Prevention using Analytics and Cybernetics Elements" (SPACE) project, a learning health system to better understand and prevent suicide incidents. We were approached and funded by the Lancashire and South Cumbria (L\&SC) Integrated Care System (ICS), part of the UK's NHS, to design and develop a learning health system that can help L\&SC ICS to determine trends and themes gathered through real-time, non-intrusive surveillance in order to prevent future suicide attempts and incidents.

The original system for the SPACE project was co-designed with the ICS through a series of consultations with people with lived-experience of suicide ideation and mental healthcare professionals. This project focused on requirements for data collection and presenting data to mental healthcare professionals. Much of the interest in the original system design was in the logic behind the system components (e.g. what data to collect, or the design of the database queries and visualizations) for detection of service-users' low moods, rather than the architecture itself. This paper, however, takes a step further by proposing a software architecture designed to support better intervention within mental health systems based on the feedback received during our consultation with different stakeholders.

The SPACE project is exploring what data can be collected from mobile phones to determine if a person is experiencing low-mood or suicide related events. This ongoing work is exploring which data to collect. For example, this includes application usage, location events near high-risk locations, screen usage patterns, and more. The original architecture collects data from a service-user's phone, using a bespoke app, and stores the data in a time series database (which is optimized for storing and querying time series data). This data can then be queried through a dashboard interface for the healthcare professionals, which would show visualizations such as graphs or the classic traffic light visualizations.

\section{B. Requirements informed by stakeholders}

We consulted 11 people with lived-experience of suicide ideation (age range between 18 and 55, male $\mathrm{n}=6$ ), and 6 mental healthcare professionals, who happened to be all female, in 2 separate workshops to better understand how to design the SPACE learning health system. The workshops were audio recorded, and transcribed verbatim with participants' consent. The qualitative data, collected in these workshops, was analyzed by 2 members of the research team using Braun and Clarke thematic analysis [44] to identify overarching themes from this consultation. The analysis was inductive and, therefore, data driven. We append quotes provided with participant numbers, and an indication of whether quotes are from lived-experience participants (e.g, P1) or mental healthcare professionals (e.g, HP1).

\section{Ethics}

We received ethical approval from Lancaster University's research ethics committee. We collaborated with 2 mental healthcare professionals (2MHP) from the ICS, who were responsible for ensuring risks to individuals were mitigated. 2MHP provided additional ethical oversight of the ethics application, study design, recruitment, communication (video, consent forms), design and approval of workshop activities, and overseeing interactions and wellbeing of the study participants (ensuring duty of care) during and after the workshops. Recruitment of participants for both workshops was directly handled by $2 \mathrm{MHP}$.

\section{Findings}

We set out to find best approaches for detecting changes in mental wellbeing through our learning health system, however, this consultation opened our eyes to the intervention element of the work. Through this consultation, participants shared important concepts related to how they wished to be supported and their preferred methods of intervention. This led to the accidental discovery of the value of peer support. In the following we expand on these values by discussing the 6 overarching themes developed from our workshops: (1) social isolation; (2) lack of resources; (3) risk assessment; (4) discontinuity of care; (5) social connectedness; (6) invaluable peer support.

1) Social Isolation: Participants with lived-experience of suicide ideation shared how they feel "alone, so alone, didn't feel I could trust anyone" (P6) and that "no one would really notice if I wasn't here" (P10). P3 expanded on loneliness and expressed this feeling as "more the feeling of having nobody 
to identify with or be understood by, abandonment: people not caring", and shared her coping strategy as "not bottling up: engage with people/services/hobbies and distractions". Health professionals also highlighted isolation as a major issue: "increase in anxiety which in a community setting may lead to more isolation, not leaving their home, not seeing others, not getting shopping” (HP6). Similarly, P5 explained how "being part of something" (P5) made him feel happy, and how "connection with others" (P5) helped him with feeling less distressed. As a strategy for managing low moods, P10 explained how he would try to "get surrounded by positive people so when the dark thoughts come I can try control them". Engagement with others and trying not to isolate themselves was a strategy suggested by all participants: "help others and connect with the right kind of people" (P8). Health professionals echoed the need for having a role and a purpose as a useful way of feeling better connected socially " $I$ guess children growing up and being less dependent and then retiring, it's massive isn't it in terms of loss of role" (HP4).

2) Lack of Resources: In discussions around the care delivery model for people who are detected as in need of support, by the learning health system, lack of resources was identified as the main barrier for providing this support: "mental health community services are very much in crisis, people are just sat on a caseload and just bubbling up in crisis, so you're just fighting fire" (HP5). This increase in demand on services was seen as a barrier for provision of high quality care: "you're not able to actually do any productive work with anyone, and so because of that obviously it's very much a second rate service. Well if people are going to feel like they're not getting support so why would they bother to contact" (HP3). With regards to the use of digital phenotyping to inform health professionals, they raised concerns about limited capacity to take actions on any detected service users in need of support: "another concern of mine with that information is around if it is coming to a crisis team and it's just information on a system and we are already saying we are absolutely overstretched as a service, we don't want to be then saying well we didn't check the data" (HP4). There was a strong emphasis on considering the capacity of services when designing supportive tools: "we had 37 people waiting for one bed, so can you imagine the increase of people being brought into the services, be great if people could be nursed at home by having like an app or something like that" (HP1). The point here is that there needs to be a series of dedicated support mechanisms as alternatives to professional services.

3) Risk Threshold: Identifying risk level is a common practice in assessment sessions, which helps health professionals in choosing the most appropriate support and treatment. Health professionals described low risk as somebody who is actively expressing suicidal thoughts and intentions but is seeking help: "contacting multiple services and has an expression that they want to get better" (HP1). Moderate risk was described as typically not seeking help but having the willingness to recover: "first time presentation, major life changes which could be in terms of employment, relationships, finances, they have a lack of support and potentially not seeking help but again I guess that thing of wanting to improve the situation" (HP1). Whereas severe risk would be somebody who has "plans in place to complete, no interest in accessing help or support, feeling helpless, hopeless, worthless, guilty, all those kinds of things. Having the access and means and lack of a protective factor [e.g., dependents to look after, such as children]" (HP1). The variation in desire to reach out and receive support is a common denominator across these three levels of risk. While health professionals highlighted the importance of supporting low and moderate risk groups: "I think it's getting people before they get to crisis isn't it, so is this app best to support primary care, low level people in terms of that engagement and working with [other] services" (HP3), the pressure on services could result in not addressing the needs of these groups regardless of their effort in seeking help. They also recognized that "everybody has different threshold to risk" (HP4), and highlighted the need for "knowing the service user that you are working with". This would require regular contact with the service user, which in the current model of care is less likely to happen, especially with low/moderate risk groups.

4) Discontinuity of Care: There were many discussions around the discontinuity of a care circle surrounding the service user. This was related to service users either receiving inpatient care, when at high risk, or that they would be discharged to go home: "They sent me home in the care of my grandparents who are 87 and 91, one of them's got dementia, the other one just had a knee replacement, they're not in any fit state to look after me" (P7). Participants acknowledged the benefit of having multiple sources of support around the service user and joining the dots to create a better care ecosystem for the service user: "I think when you've got a team of services around somebody, that somebody has got far more chance that one of the services will help them, as opposed to having just one professional in their life offering support, I think if you've got say six or seven, they've then got six or seven branches to reach out and grab a hold of" (P2). Health professionals also backed up this idea by proposing to offer a number of support options in intermediate care, for example: "for me, within this app, digital service that you're looking at providing, it would be looking at giving alternative advice lines, with multiple choice options to respond to and then looking at what's going on for that individual 'cos it's very different for every individual in terms of what they're experiencing and what their perception of need is" (HP3).

5) Social Connectedness: Participants highlighted the importance of connectivity and how receiving advice from people in similar situations could be beneficial: "once you put the substance down or anything that you used as a crutch, connectivity is the key in it, I mean being in places like this, getting a better understanding, without others I wouldn't have been able to identify where I were coming from, what I'm saying, so being able to be open to the possibility of someone being able to tell me something and I can actually hear it, rather than someone telling me something and it just going over me head all the time 'cos I can't connect with it" (P10). 
The discussions led into the topic of face-to-face support and how this model is overlooked in the design of technology enabled support for mental health. For example P2 questioned: "how did we interact with people before all this technology?" (P8) which was answered by "spoke to their faces" (P4) and "we understood body language" (P1) and "emotions" (P7). They raised a vivid gap in the direction of digital support: "no emotion in messages" (P1) and "Well there's emojis [laughter]" (P7). They saw the value in learning from each other "we were sat here today, we've all grown in some kind of manner" (P2). While they preferred face-to-face support, the idea of digital support also received positive feedback: " $a$ friend of mine's just created [a group on Facebook] called Talk Free, and people go to that group just to express how they're feeling at that moment in their mental health and there's so much support for people. I find just reading that when I'm feeling a bit shitty, that kind of picks you up a little bit, not reading other people's suffering but reading the support messages that go with it, now that's good" (P5).

6) Invaluable Peer Support: Participants all recognized the value of peer support, and had the sense that it has the potential to be of value to someone: "you're always somebody's someone. Even if it's just that you make them smile or you do something nice" (P5). In the words of P7, the support coming from peers, at any level, is powerful: "even if you can't be that person to rescue them, if you can prove that you're just there in the background if they need you". This relates to the loneliness issues previously raised by the participants, by knowing "you're there if something happens, they might not talk to you but they know if they needed you're there" (P3). In this sense, participants saw benefit in having a "walk in centre" where individuals can speak to someone about their problems without making any appointments. However, they acknowledged the high demands on services, and suggested to make use of peer support groups: "I think that's where peer to peer support comes in and I think that's really useful" (P4). They provided examples as to how peer support models in a small scale are effective: "it's so important that people see what happens within [our peer group] as we have people that come to a group who are struggling in that moment, and they'll leave knowing that they've had that conversation with somebody who's like minded, they can identify with what they're going through, they've given them some kind of advice and some kind of reassurance to say you're not on your own" (P1). The notion of facilitating peer support through the proposed learning health system received positive feedback "that is absolutely the way forward, I can't understand anybody saying no to that" (P5) and was seen as a way to "take pressure off services a little bit" (P1). They compared an informal peer support model to mental health chat groups available on social media where "there's likeminded people, and somebody can then reach out to you and offer you that branch" (P7). They expressed how offering this connectivity both in face-to-face and digital models such as "cyber friends" (P5) would encourage them to engage with the proposed learning health system.

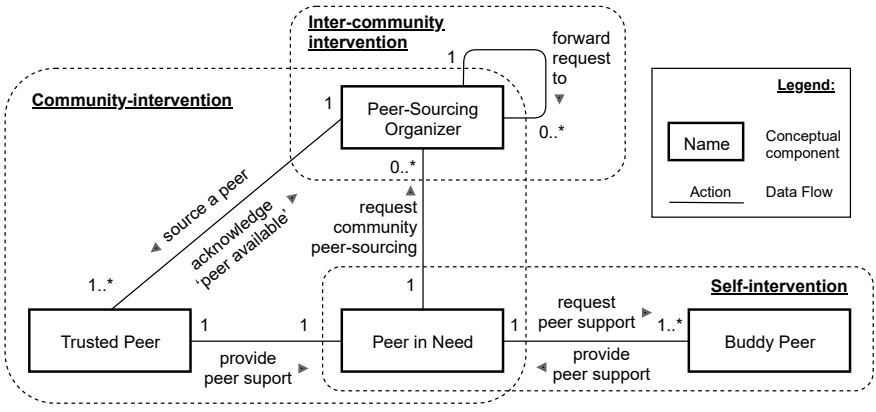

Fig. 1. Proposed Software Architecture for Peer-Sourcing in Mental Health.

\section{E. Derived Software Requirements}

Based on the findings explained in Section III-D, we have derived software requirements of a system utilizing peersupport in mental health. These are explained below. Each of the requirements are presented with their connections to specific findings from the workshops:

R1: Detect changes in patterns of behaviors/moods: The system shall detect changes in patterns of behaviors/moods of a service user and, once detected, it shall start sourcing the available support (linked to III-DI- Social Isolation and III-D3 - Risk Threshold). Common symptoms of social isolation include the lack of interaction with others (P6 and P10 in III-D1) and staying at home for a longer period of time (HP6 and P5 in III-D1). These symptoms are not usually picked up by health professionals, if they are considered to be low/moderate risks (HP1 in III-D3). For those in the low/moderate groups, it is important that the system recognizes such symptoms and starts intervening when a user has 'dark thoughts' at an early stage (HP3 and HP3 in III-D3).

R2: Registration of preferred peers to be contacted: A user shall be able to register a set of immediate contactable peers and a set of communities where the user belongs to (linked to III-D5-Social Connectedness). It is critical that help should first come from a closely connected peer or a group which is well-connected to the service user (P10 and P5 in III-D5). A message or help from unfamiliar persons or groups could be easily heard vacantly and ignored (P10 in III-D5). Therefore, the user should be able to explicitly specify the direct peers (i.e., buddy peers) and well-known communities, in which the leader of the community, can find 'trusted peers' for the user.

R3: Autonomous sourcing of available peers: The system shall start looking for available peers to intervene when changes in patterns of behaviors/moods are detected (linked to III-D4 - Discontinuity of Care. Users might ask for help from their peers by themselves, but only if they were aware of the situation that they were at stake. In most cases, however, it can be difficult for service users to know when to ask for help (HP3 in III-D4). As such the system should have a capability that it starts asking for help through peer-sourcing autonomously. 
R4: Dynamic change of peer-sourcing scope: The system shall be able to change the scope of target source, where an available peer would be searched (linked to III-D6 Invaluable Peer Support). As pointed out by P7 in III-D6. the help should be sourced from all available levels, including friends, local community members and health professionals. It means that the system should be able to search different sources in an orderly manner. To address this need, we propose the following three different levels:

R4.1: Self-intervention level: a peer shall be sourced from a pre-defined list of peers (i.e., buddy peers, like family or friends) by the user;

R4.2: Community-intervention level: a peer shall be sourced from a community where the user belongs to and the sourcing shall be performed by the leader of the community;

R4.3: Inter-community-intervention level: a peer shall be sourced from other communities, which include other user communities and professional organizations (e.g., charities, National Health Service (NHS), police department, etc.)

R5: Selection of sources: The peer-sourcing shall begin with the 'Self-intervention' level and then 'Communityintervention' and finally it shall be performed at the 'Inter-community-intervention' level (linked to III-D2 Lack of Resources. As all participating health professionals pointed out in III-D2, lack of resources was the main barrier for providing mental health services. This means that low/medium risk groups might not have opportunities to receive help when needed due to the high demand for mental health professionals. R5 is aiming to alleviate such difficulties by explicitly distributing and ordering the service sources from close/local peer groups to professional groups.

In the following section, we explain how we revised the current software architecture to deliver these requirements.

\section{REFInEd SOFTwARE ARCHITECTURE}

During the process of deriving requirements from the findings of the workshops, it became evident that we needed a new software architecture to support the peer-sourcing from varying sources. Whilst the crowd-sourcing frameworks are adequate for centralized and homogeneous task sourcing environments, and the current architecture would work for a clinical decision support system, they could not deliver all the requirements listed in Section III-E. In the following, we propose a peer-sourcing architecture and show an instance of it for a mental-health support system.

\section{A. An Architecture Style for Peer-Sourcing: A Meta Model}

Figure 1 is a conceptual view of the proposed peer-sourcing architecture meta-model and the name of the conceptual components represents its main role in the architecture. Each component is explained in the following:

Peer in Need (PN): As the name suggests, this component represents an individual who would need the support provided

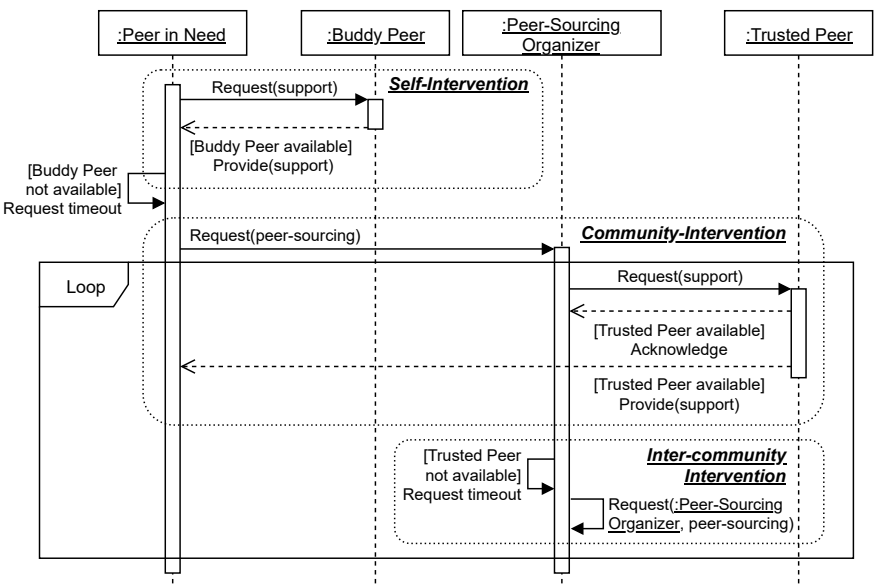

Fig. 2. Sequence Diagram of Peer-Sourcing Architecture Model.

by its peers (i.e., a service user). The main functionalities allocated to this component include ' $\mathrm{R} 1$ : detect changes in patterns of behaviors/moods' and 'R2: registration of preferred peers to be contacted'. The detection described in R1 triggers the peer-sourcing: once detected, this component first reaches out for its close/familiar peers (i.e., Buddy Peers) for their direct supports. If no buddy peers are available and capable of providing support, a leader of a participating community (i.e., Peer-sourcing Organizer) is contacted to source available peers in the community (i.e., Trusted Peer). (Related requirements: $R 1$ and $R 2$ )

Buddy Peer (BP): A service user can decide whom should be contacted when changes in patterns of behavior/moods are detected. A group of peers that should be contacted with the highest priority is represented by this component (i.e., BP) and may include family members and/or close friends. The PN maintains a list of BPs and searches for support from the members in this group. This component should be able to warn the registered BPs with proper information that can show the status of the service user. When the service user can find a peer in the BP group, this completes the 'Self-intervention' scope. (Related requirements: $R 3, R 4.1$ and $R 5$ )

Peer-Sourcing Organizer (PSO): A user can also decide a community leader whom should be contacted when no buddy peers are available. The PSO component represents community leaders and maintains a list of members of the community to be contacted (i.e., Trusted Peer). If a PSO can find an available trusted peer to support the service user, this completes the 'Community Intervention' scope. If no trusted peers are available, the request should be forwarded to a PSO in a higher level (e.g., Mental Health Charity Leader, NHS Support Group Leader, etc.): this propagation of support request to outside of the community is called 'Inter-community Intervention'. (Related requirements: $R 3, R 4.2, R 4.3$ and $R 5$ )

Trusted Peer (TP): This component represents a member of a community where the user is participating. For example, a member of local mental health group can be a trusted peer and be contacted by a community leader (i.e., a PSO) to support 
a peer in need. If a TP is available, it should acknowledge its availability to the PSO and provide a peer support. The TP component should also be able to warn the registered trusted peers with the status of the service user. (Related requirement: R4.2)

To illustrate the interaction of these conceptual components to deliver the requirements in Section III-E, we used a sequence diagram of the peer-sourcing architecture, shown in Figure 2. The Figure shows that the initial peer-sourcing is performed at the self-intervention level and then the sourcing is carried out at the community-interventions level, when no buddy peer is available (see the Request timeout message at the timeline of Peer in Need. The 'Loop' block captures the idea of propagation of peer-sourcing to other communities, if Peer in Need could not receive a Provide(support) message.

In this section we have explained the roles and major functionalities of the conceptual components in the peersourcing software architecture. Based on this model, we have derived an instance of it for our SPACE project. This instance architecture model is explained in the next section.

\section{B. An instance of the Peer-Sourcing Architecture}

The conceptual components in Figure 1 capture all participating entities to realize the peer-sourcing requirements. In this section, we demonstrate its feasibility by instantiating a software architecture to deliver peer-sourcing in the context of suicide prevention support.

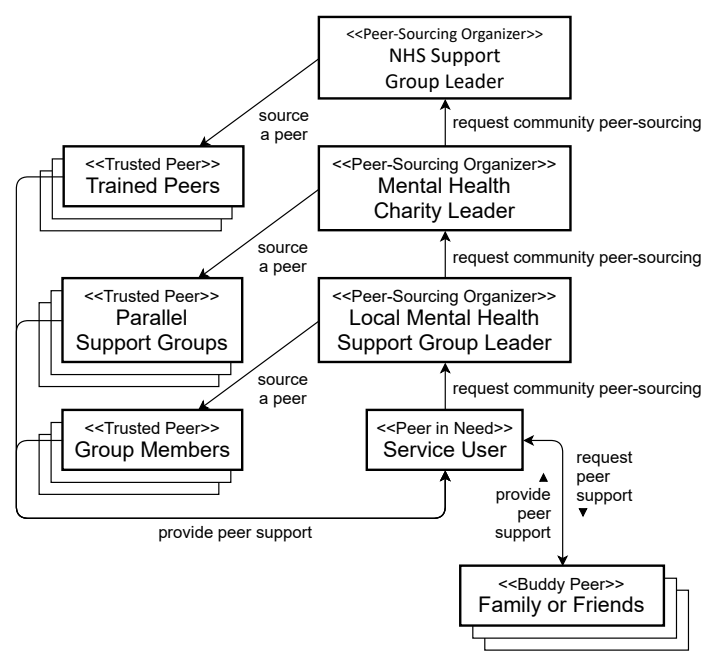

Fig. 3. An Instance of the Proposed Architecture in Figure 1

Figure 3 depicts an instance of the software architecture. The Service User (Peer in Need) component monitors the status of a service user and triggers a support request to the Family or Friends (Buddy Peer) components for the self-intervention. If this is not successful, Service User requests community peer-sourcing from the Local Mental Health Support Group Leader (Peer-Sourcing Organizer) component for the community intervention. If these two different levels of intervention attempts also cannot provide support, Local Mental Health Support Group Leader forwards the request to
Mental Health Charity Leader, which is an instance of PeerSourcing Organizer at a higher level. This dynamic scoping of peer-sourcing continues until a peer can be found from these different sources (e.g., family, friends, local community, charity, professional organization)

\section{DiscusSION}

In this section, we discuss the proposed architecture in terms of additional quality requirements, challenges and limitations.

Timing Requirements. Though the response time requirements of peer-sourcing is not (hard-) real time, it is expected that a peer should be found within a reasonable time frame. For example, some quotes from the workshops related to the response time requirements include: "if the data was fed to a crisis team then instant access would be needed" and "instantly, changes in behavior predicted to be of high risk nature, could be alerts". Therefore, the detailed design of the conceptual components of the peer-sourcing architecture should be analyzed and tested to meet the time constraints.

Dynamic Scoping of Peer-Souring. The sources for peersourcing is distributed by nature and cannot be confined to a single group, community, charity or professional organization. At the same time, the sourcing should be performed in an orderly manner, starting from self-intervention to communityintervention and finally inter-community intervention levels. Moreover, the decision to move on from one source to others can only be decided at runtime, considering the available peers at that particular moment in time. We have addressed this requirement by explicitly separating the class of sources (i.e., Buddy Peer and Trusted Peer) and propagating the scope of sources via the hierarchy of Peer-Sourcing Organizer.

Peer Selection and Task Assignment. Similar to the worker selection and task assignment problem in crowd-sourcing, peer-sourcing systems also need to contain the components that can match the peer support tasks with appropriate peer workers. However, it is more complicated in the context of peer-sourcing than crowd-sourcing due to more subtle factors. For example, crowd-sourcing systems usually consider the expertise for worker selection, but peers who seek help may prefer peers with similar life experiences and empathy, which is hard to be measured and embedded as a match component into the system. Another exemplary element is that, in peer support, the ability to self-express and comfort others is also an important component of expertise. However, these attributes are hard to be qualified and further taken into account in the optimization of peer selection or task assignment. Finding representational models for such attributes and components and developing algorithms for their estimation will be critical in enabling the peer-sourcing approach. Besides, in contrast to crowd-sourcing tasks which mainly use monetary incentives to motivate workers to participate, peer supporters have extra incentive to stay well if he or she feels to be a role model for others. How to reflect these characteristics of peer-sourcing into the software architecture is still worth studying. The current version of our proposed architecture provides a highlevel framework - a first step in this new research direction. 
Privacy Preserving Function. The advantage of online peer support is seen in the anonymity and privacy it affords to both the person seeking support and the peer supporter [45]. Involving recovered peers as peer supporters in a face-to-face setting is not a new concept and has been shown to be effective for decades [46]. While there exists concerns over breaking confidentiality in this model [47], Davidson et al. highlighted that there is no reason to believe that preserving privacy will be any more difficult for peer supporters than for anyone else [48]. In fact, "peer supporters" (Buddy Peer, Trusted Peer), based on their own personal experiences as service users, are more likely to protect their peer's privacy even more so than non-peer staff (e.g., health professionals) [48]. Alternatively, Kemp and Henderson proposed training about boundaries and ethical issues as a key solution for maintaining privacy in peer support model [49]. Although this requirement is not addressed in our proposed architecture, investigating the role of training to embed and preserve privacy in peer-sourcing architecture will be essential future work.

Value Creation. The crowd in crowd-sourcing receives a direct recompense for performing the task [50] and the direct benefit for the Trusted Peer in the peer-sourcing model also needs to be considered.

While improvements in social and occupational functionality of the Trusted Peer are seen as direct benefits [20], Trusted Peers need to be treated like workers in order for the proposed system to be sustainable. This is particularly important as Trusted Peers need to treat the task at hand as a service commitment. It needs to be clear what the Trusted Peer gets in return and how they get financially supported and sustained. Financial reward could be received for the completion of a peer-support session or improved measures in outcome for a Peer-in-Need (for example, the reduction in number of visits to traditional services over a predefined time frame). "High demands on services" was raised repeatedly in our workshop, and participants see peer-support as a way to "take pressure off services", for example saving money and reducing direct staff time. These savings could provide additional resource to run a peer-sourcing service. This requires further research to investigate how a financial model could be ethically integrated into a peer-sourcing model.

Evaluation of the Proposed Software Architecture. The main focus and contributions of this paper are in analyzing/deriving requirements from various stakeholders in a real mental health care context and designing a software architecture to address the requirements with peer-sourcing. The architecture model was validated through developing its instance for our project and executing the scenarios from the workshops, however, we still need to further verify the model via implementing and deploying a system in a real environment. For example, timing requirements for different cases (e.g., from low/medium risk cases to urgent/emergent cases) should be carefully collected and tested with different stakeholders.

\section{CONCLUSiON}

The current practice and care pathway, consisting of monitoring of service users' symptoms and facilitating an intervention when unexpected changes from routine patterns are recognized, is unsustainable. It is heavily dependent on the availability of health professionals, which is scarce in the mental health care domain.

Through a mental health research project in which we engaged with various stakeholders from a real mental health care context, we learned that an appropriate source of support needs to take into account user's preferences as in who and when to engage with, in their care and specifically the preference of peer support which is increasingly on demand. In response, and to provide a systematized support for this rising need, we have proposed the software architecture to utilize peer support via peer-sourcing of potential support providers from various groups. We presented requirements analysis based on a study with service users and health professionals. We also demonstrated the architecture feasibility by instantiating the model in our project context and executing the user scenarios gathered from the workshop activities.

In future work, we will implement and deploy an instance of the proposed architecture with more practical factors (e.g., timing constraints, peer selection and privacy preservation) and collect feedback from service users and health professionals to inform refinements and further assessments.

\section{ACKNOWLEDGMENT}

We thank all participants from the SPACE project workshops and also our collaborators in the Lancashire and South Cumbria ICS, and National Health Service Organization.

\section{REFERENCES}

[1] Our World in Data. (2017) Share of population with mental health and substance use disorders. [Online]. Available: https://ourworldindata.org/ grapher/share-with-mental-and-substance-disorders

[2] S. Abdullah and T. Choudhury, "Sensing technologies for monitoring serious mental illnesses," IEEE MultiMedia, vol. 25, no. 1, pp. 61-75, 2018.

[3] R. Morriss, I. Vinjamuri, M. A. Faizal, C. A. Bolton, and J. P. McCarthy, "Training to recognise the early signs of recurrence in schizophrenia," Cochrane Database of Systematic Reviews, no. 2, 2013.

[4] I. Barnett, J. Torous, P. Staples, L. Sandoval, M. Keshavan, and J.-P. Onnela, "Relapse prediction in schizophrenia through digital phenotyping: a pilot study," Neuropsychopharmacology, vol. 43, no. 8, p. 1660 , 2018.

[5] M. Hassanalieragh, A. Page, T. Soyata, G. Sharma, M. Aktas, G. Mateos, B. Kantarci, and S. Andreescu, "Health monitoring and management using internet-of-things (iot) sensing with cloud-based processing: Opportunities and challenges," in 2015 IEEE International Conference on Services Computing. IEEE, 2015, pp. 285-292.

[6] C.-L. Dennis, "Peer support within a health care context: a concept analysis," International journal of nursing studies, vol. 40, no. 3, pp. 321-332, 2003

[7] L. Davidson, M. Chinman, D. Sells, and M. Rowe, "Peer support among adults with serious mental illness: a report from the field," Schizophrenia bulletin, vol. 32, no. 3, pp. 443-450, 2006.

[8] J. Huber, T. Muck, P. Maatz, B. Keck, P. Enders, I. Maatouk, and A. Ihrig, "Face-to-face vs. online peer support groups for prostate cancer: a cross-sectional comparison study," Journal of Cancer Survivorship, vol. 12, no. 1, pp. 1-9, 2018.

[9] J. Naslund, K. Aschbrenner, L. Marsch, and S. Bartels, "The future of mental health care: peer-to-peer support and social media," Epidemiology and psychiatric sciences, vol. 25, no. 2, pp. 113-122, 2016. 
[10] I. Caramlau, J. Barlow, S. Sembi, K. McKenzie-McHarg, and C. McCabe, "Mums 4 mums: structured telephone peer-support for women experiencing postnatal depression. pilot and exploratory ret of its clinical and cost effectiveness," Trials, vol. 12, no. 1, p. 88, 2011.

[11] A. E. Dam, M. E. de Vugt, I. P. Klinkenberg, F. R. Verhey, and M. P. van Boxtel, "A systematic review of social support interventions for caregivers of people with dementia: are they doing what they promise?" Maturitas, vol. 85, pp. 117-130, 2016.

[12] D. C. Mohr, J. Duffecy, J. Ho, M. Kwasny, X. Cai, M. N. Burns, and M. Begale, "A randomized controlled trial evaluating a manualized telecoaching protocol for improving adherence to a web-based intervention for the treatment of depression," PloS one, vol. 8, no. 8, p. e70086, 2013.

[13] S. Mead and C. MacNeil, "Peer support: What makes it unique," International Journal of Psychosocial Rehabilitation, vol. 10, no. 2, pp. 29-37, 2006.

[14] K. O'Leary, S. M. Schueller, J. O. Wobbrock, and W. Pratt, ““'suddenly, we got to become therapists for each other": Designing peer support chats for mental health," in Proceedings of the 2018 CHI Conference on Human Factors in Computing Systems, ser. CHI '18. New York, NY, USA: ACM, 2018, pp. 331:1-331:14.

[15] J. Repper and T. Carter, "A review of the literature on peer support in mental health services," Journal of mental health, vol. 20, no. 4, pp. 392-411, 2011.

[16] B. Lloyd-Evans, E. Mayo-Wilson, B. Harrison, H. Istead, E. Brown, S. Pilling, S. Johnson, and T. Kendall, "A systematic review and metaanalysis of randomised controlled trials of peer support for people with severe mental illness," BMC psychiatry, vol. 14, no. 1, p. 39, 2014.

[17] I. D. Goldstrom, J. Campbell, J. A. Rogers, D. B. Lambert, B. Blacklow, M. J. Henderson, and R. W. Manderscheid, "National estimates for mental health mutual support groups, self-help organizations, and consumeroperated services," Administration and Policy in Mental Health and Mental Health Services Research, vol. 33, no. 1, pp. 92-103, 2006.

[18] S. L. Bernecker, K. Banschback, G. D. Santorelli, and M. J. Constantino, "A web-disseminated self-help and peer support program could fill gaps in mental health care: Lessons from a consumer survey," JMIR mental health, vol. 4, no. 1, p. e5, 2017.

[19] P. Bracke, W. Christiaens, and M. Verhaeghe, "Self-esteem, self-efficacy, and the balance of peer support among persons with chronic mental health problems," Journal of Applied Social Psychology, vol. 38, no. 2, pp. 436-459, 2008.

[20] M. S. Salzer, N. Darr, G. Calhoun, W. Boyer, R. E. Loss, J. Goessel, E. Schwenk, and E. Brusilovskiy, "Benefits of working as a certified peer specialist: Results from a statewide survey." Psychiatric Rehabilitation Journal, vol. 36, no. 3, p. 219, 2013

[21] A. Barak, "Emotional support and suicide prevention through the internet: A field project report," Computers in Human Behavior, vol. 23 , no. 2, pp. 971-984, 2007.

[22] R. R. Morris, S. M. Schueller, and R. W. Picard, "Efficacy of a webbased, crowdsourced peer-to-peer cognitive reappraisal platform for depression: randomized controlled trial," Journal of medical Internet research, vol. 17, no. 3, p. e72, 2015.

[23] G. Tartarisco, G. Baldus, D. Corda, R. Raso, A. Arnao, M. Ferro, A. Gaggioli, and G. Pioggia, "Personal health system architecture for stress monitoring and support to clinical decisions," Computer Сотmиnications, vol. 35, no. 11, pp. 1296 - 1305, 2012.

[24] A. Mouttham, L. Peyton, B. Eze, and A. Saddik, "Event-driven data integration for personal health monitoring," Journal of Emerging Technologies in Web Intelligence, vol. 1, no. 2, pp. 110-118, 2009.

[25] F. Velickovski, L. Ceccaroni, J. Roca, F. Burgos, J. B. Galdiz, N. Marina, and M. Lluch-Ariet, "Clinical decision support systems (cdss) for preventive management of copd patients," Journal of translational medicine, vol. 12, no. 2, p. S9, 2014.

[26] S. H. El-Sappagh and S. El-Masri, "A distributed clinical decision support system architecture," Journal of King Saud University - Computer and Information Sciences, vol. 26, no. 1, pp. 69 - 78, 2014.

[27] A. Frame, M. LaMantia, B. B. R. Bynagari, P. Dexter, and M. Boustani, "Development and implementation of an electronic decision support to manage the health of a high-risk population: the enhanced electronic medical record aging brain care software (emr-abc)," EGEMS, vol. 1, no. 1, 2013.

[28] J. Dunkel, A. Fernández, R. Ortiz, and S. Ossowski, "Event-driven architecture for decision support in traffic management systems," Expert Systems with Applications, vol. 38, no. 6, pp. 6530 - 6539, 2011.
[29] A. Hein, M. Eichelberg, O. Nee, A. Schulz, A. Helmer, and M. Lipprandt, "A service oriented platform for health services and ambient assisted living," in 2009 International Conference on Advanced Information Networking and Applications Workshops. New York, NY, USA: IEEE, 2009, pp. 531-537.

[30] T. Alahäivälä, H. Oinas-Kukkonen, and T. Jokelainen, "Software architecture design for health bcss: Case onnikka," in Persuasive Technology, S. Berkovsky and J. Freyne, Eds. Berlin, Heidelberg: Springer Berlin Heidelberg, 2013, pp. 3-14.

[31] C. Bull, D. Asfiandy, A. Gledson, J. Mellor, S. Couth, G. Stringer, P. Rayson, A. Sutcliffe, J. Keane, X. Zeng, A. Burns, I. Leroi, C. Ballard, and P. Sawyer, "Combining data mining and text mining for detection of early stage dementia: the sams framework," in Proceedings of LREC 2016 Workshop on Resources and Processing of Linguistic and ExtraLinguistic Data from People with Various Forms of Cognitive/Psychiatric Impairments (RaPID'16). Paris, France: ELRA, 2016, pp. 35-40.

[32] J. Howe, "The rise of crowdsourcing," Wired magazine, vol. 14, no. 6 , pp. 1-4, 2006.

[33] K. Mao, L. Capra, M. Harman, and Y. Jia, "A survey of the use of crowdsourcing in software engineering," Journal of Systems and Software, vol. 126, pp. 57-84, 2017.

[34] G. Xintong, W. Hongzhi, Y. Song, and G. Hong, "Brief survey of crowdsourcing for data mining," Expert Systems with Applications, vol. 41, no. 17, pp. 7987-7994, 2014.

[35] H. Paulheim, "Knowledge graph refinement: A survey of approaches and evaluation methods," Semantic web, vol. 8, no. 3, pp. 489-508, 2017.

[36] C. Kulkarni, S. P. Dow, and S. R. Klemmer, "Early and repeated exposure to examples improves creative work," in Design thinking research. Springer, 2014, pp. 49-62.

[37] E. Mollick, "The dynamics of crowdfunding: An exploratory study," Journal of business venturing, vol. 29, no. 1, pp. 1-16, 2014.

[38] R. Simpson, K. R. Page, and D. De Roure, "Zooniverse: observing the world's largest citizen science platform," in Proceedings of the 23rd international conference on world wide web. ACM, 2014, pp. 10491054.

[39] G. Paolacci, J. Chandler, and P. G. Ipeirotis, "Running experiments on amazon mechanical turk," Judgment and Decision making, vol. 5, no. 5, pp. 411-419, 2010.

[40] M.-R. Ra, B. Liu, T. F. La Porta, and R. Govindan, "Medusa: A programming framework for crowd-sensing applications," in Proceedings of the 10th international conference on Mobile systems, applications, and services. ACM, 2012, pp. 337-350.

[41] J. Wang, Y. Wang, L. Wang, and Y. He, "Gp-selector: a generic participant selection framework for mobile crowdsourcing systems," World Wide Web, vol. 21, no. 3, pp. 759-782, 2018.

[42] H. Xiong, Y. Huang, L. E. Barnes, and M. S. Gerber, "Sensus: a crossplatform, general-purpose system for mobile crowdsensing in humansubject studies," in Proceedings of the 2016 ACM International Joint Conference on Pervasive and Ubiquitous Computing. ACM, 2016, pp. 415-426.

[43] D. M. Aanensen, D. M. Huntley, E. J. Feil, B. G. Spratt et al., "Epicollect: linking smartphones to web applications for epidemiology, ecology and community data collection," PloS one, vol. 4, no. 9, p. e6968, 2009.

[44] V. Braun and V. Clarke, "Using thematic analysis in psychology," Qualitative research in psychology, vol. 3, no. 2, pp. 77-101, 2006.

[45] Y. Takahashi, C. Uchida, K. Miyaki, M. Sakai, T. Shimbo, and T. Nakayama, "Potential benefits and harms of a peer support social network service on the internet for people with depressive tendencies: qualitative content analysis and social network analysis," Journal of medical Internet research, vol. 11, no. 3, p. e29, 2009.

[46] L. Davidson, J. Rakfeldt, and J. Strauss, The roots of the recovery movement in psychiatry: Lessons learned. John Wiley \& Sons, 2011.

[47] H. Cowie and P. Wallace, Peer support in action: From bystanding to standing by. Sage, 2000.

[48] L. Davidson, C. Bellamy, K. Guy, and R. Miller, "Peer support among persons with severe mental illnesses: a review of evidence and experience," World psychiatry, vol. 11, no. 2, pp. 123-128, 2012.

[49] V. Kemp and A. R. Henderson, "Challenges faced by mental health peer support workers: Peer support from the peer supporter's point of view." Psychiatric Rehabilitation Journal, vol. 35, no. 4, p. 337, 2012.

[50] F. Kleemann, G. G. Voß, and K. Rieder, "Un(der)paid innovators: The commercial utilization of consumer work through crowdsourcing," STI Studies, vol. 4, no. 1, p. 5, 2008. 